\title{
Are Convexity Meningiomas All the Same? A Clinico-Radiological Analysis of Surgically Treated Eloquent Areas Convexity Meningiomas
}

Giannantonio Spena 1 , Francesco Guerrini $2 \underset{2}{2}$, Paola Decet $\stackrel{4}{2}$, Federico D'agata $\stackrel{5}{2}$, Elena

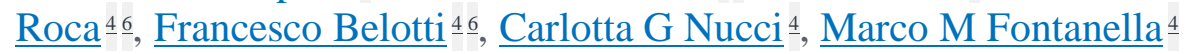

Affiliations expand

- PMID: 31298505

- DOI: $\underline{10.23736 / \mathrm{S} 0390-5616.19 .04713-1}$

\section{Abstract}

Background: Convexity meningiomas are considered low-risk tumors, with high possibility of cure and low risk of relapse after resection. Very few studies have investigated meningiomas located in or around highly eloquent regions (namely perirolandic and perisylvian fissures). This study aimed to determine the differences in preoperative characteristics and postoperative outcomes between convexity meningiomas at eloquent area and non-eloquent areas.

Methods: Retrospective study on patients who underwent surgical resection for convexity meningioma. Patients were divided into eloquent and non-eloquent area. Statistical analysis was made comparing preoperative and postoperative data of both groups.

Results: The study included a total of 117 patients: 80 with eloquent area tumor and 37 with non- eloquent area tumor. Statistically significant differences were detected between the groups in preoperative KPS (93 \pm 10 in eloquent vs. $97 \pm 6$ in non-eloquent; $p=.008$ ) and in large-caliber vein involvement $(76.3 \%$ in cases vs. $16.2 \%$ in controls; $p<.001)$. Postoperatively, patients with eloquent area tumors showed initial deterioration in neurological status followed by recovery; final outcomes were comparable to that of patients with non-eloquent area tumors. However, patients with eloquent area meningiomas had higher propensity to suffer from seizures postoperatively. Postoperative complications and long-term outcomes were not significantly different between the two groups. 
Conclusions: Patients with eloquent areas convexity meningiomas do not appear to have higher surgical risk. Neurological status is more likely to worsen immediately after surgery but long-term recovery is satisfactory. Seizure control after surgery appears to be poorer in patients with perirolandic meningioma. 